\title{
Una fundamentación "merleaupontyana" para la apuesta metodológica de Bourdieu en Argelia
}

\author{
A foundation based on "Merleau-Ponty" for Bourdieu's \\ methodological approach in Algeria \\ Juan Dukuen / juanduk2002@yahoo.com.ar \\ Consejo Nacional de Investigaciones Cientificas y Técnicas (Conicet), Argentina
}

\begin{abstract}
The purpose of this paper is to show that Bourdieu's first methodological approach to overcome Dilthey's opposition between "explain and understand" has a misrecognized basis in the remarks by Merleau-Ponty on the same topic. We will verify that this is understandable if one considers that Bourdieu was trained as a philosopher in the framework of learning oriented toward the phenomenological tradition and that such "philosophical cultural capital" works in his first methodological experimentation in the passage from philosophy to socio-anthropology in Algeria (1957-1964). This will show that although the thesis on "explain and understand" reappears in The Weight of the World 30 years later, it does not respond to a wink of accommodation to "postmodernism", but to a methodological approach that is a clear development from the beginning of Bourdieu's reflections on the statute of social sciences.
\end{abstract}

Key words: Bourdieu, Merleau-Ponty, methodology, understand, Anthropology.

Resumen: El objetivo de este artículo es mostrar que la primera apuesta de Bourdieu en el terreno metodológico, relativa a la superación de la oposición diltheyana entre "explicar y comprender", encuentra un fundamento desconocido en señalamientos de Merleau-Ponty sobre el mismo tópico. Afirmaremos que esta fundamentación se comprende si se tiene en cuenta que Bourdieu se formó como filósofo en el marco de un aprendizaje orientado hacia la tradición fenomenológica y que ese "capital cultural filosófico" opera al jugar sus primeras cartas de experimentación metodológica en el pasaje de la filosofía a la socioantropología en Argelia (1957-1964). Esto permitirá evidenciar que si bien la tesis sobre "explicar y comprender" reaparece en La Miseria del mundo treinta años después, ello no responde a un guiño de acomodamiento al "posmodernismo", sino a una apuesta metodológica que encuentra un claro desarrollo desde el comienzo de la reflexión bourdiana sobre el estatuto de las ciencias sociales.

Palabras clave: Bourdieu, Merleau-Ponty, metodología, comprender, antropología. 


\section{Introducción ${ }^{12}$}

Este trabajo parte del supuesto de que para comprender la génesis de las tomas de posición metodológicas de Bourdieu en su debut en socioantropología, ${ }^{3}$ es necesario dar cuenta, en primera instancia, del encuentro entre su formación académica en filosofía y las condiciones sociales de guerra y revolución en las que se desarrollan sus investigaciones empíricas en Argelia (1957-1964).

Si bien la relación entre la formación filosófica de Bourdieu y su experimentación teórico/metodológica en socioantropología es algo que se observa en el desarrollo de varios de los conceptos claves de la "teoría de la práctica" - ethos, hexis, eidos, habitus, esquema (scheme), entre otros- (Bourdieu, 1972, 1980; Heran, 1987; Hong Sung-Min, 1999; Martínez, 2007:31-159), no parece haber sido suficientemente trabajada en lo que concierne a ciertas apuestas metodológicas en el plano de la investigación empírica: en ese punto se muestra su deuda con las obras de Durkheim, Weber y Bachelard (Baranger, 2004), pero en mucho menor medida con una de las tradiciones filosóficas que reasume como una "caja de herramientas"; es decir, con la línea fenomenológica que va de Husserl a Merleau-Ponty (pasando, por supuesto, por Heidegger y Sartre). ${ }^{4}$

1 Este artículo cuenta con el aval de los proyectos PICT 2012-2751 (ANPCyT-Argentina) y PIP 11220100100307 (Conicet-Argentina) dirigidos por la Dra. M. Kriger.

2 En este artículo profundizo análisis desarrollados parcialmente en mi tesis doctoral (Dukuen, 2013) sobre el vínculo fundamental de la teoría de la práctica de Bourdieu con la fenomenología de Merleau-Ponty. Mi agradecimiento a Graciela Ralon de Walton por compartirme su profundo saber sobre la obra de Merleau-Ponty; a Denis Baranger y Ana Teresa Martínez que me facilitaron textos agotados o de muy difícil acceso; y a Carolina Ferrante por los sugerentes comentarios.

3 Aunque Vázquez García (2002) lo define como una etno-sociología, nosotros preferimos hablar de una socioantropología, por la importancia que tiene para Bourdieu la dignificación de la antropología hecha por Lévi-Strauss y la traducción realizada por Foucault de la $A n$ tropología de Kant; así como por la necesidad de superar las distinciones entre etnología y etnografía y la división del trabajo entre recolección de datos e interpretación de los mismos (Bourdieu, 1987).

4 Y esto porque el hecho de ubicar o no a la Teoría de la práctica de Bourdieu en el marco de la tradición fenomenológica es objeto de disputa tanto entre los bourdianos, como entre los críticos que operan desde una sociología de orientación fenomenológica. Sobre esta disputa, véase: Sapiro (2007a, 2007b), Throop y Murphy (2002), Bourdieu (2002), Haber (2004), Bimbenet (2006, 2011), García (2008), Ralon de Walton (2010), Belvedere (2011a, 2011 b, 2012) y Dukuen (2010, 2011a, 2011b, 2012, 2013). 
En este artículo vamos a señalar que ese "capital cultural filosófico" vuelto disposiciones, libido sciendi, opera en el momento en que Bourdieu juega sus primeras cartas de experimentación metodológica en su pasaje iniciático de la filosofía a la socioantropología en Argelia (1957-1964). En ese sentido argumentaremos que la primera apuesta fuerte de Bourdieu en el terreno metodológico relativa a superación de la oposición diltheyana entre "explicar y comprender" (Bourdieu et al., 1963), encuentra un fundamento desconocido (méconnu) 5 en algunos señalamientos de Merleau-Ponty (1945) sobre el mismo tópico. Afirmaremos que la fundamentación ${ }^{6}$ merleaupontyana se comprende si se tiene en cuenta que Bourdieu se formó como filósofo en el marco de un aprendizaje orientado a la tradición fenomenológica que incluye a Husserl, Heidegger, Merleau-Ponty y Sartre. Esto permitirá evidenciar, de pasada, que si bien esta tesis reaparece con claridad en el acápite "Comprender" de La Miseria del mundo, treinta años después (Bourdieu, 1993: 910), no lo hace como un guiño de acomodamiento al posmodernismo, sino como una apuesta metodológica que encuentra un claro desarrollo desde el comienzo de la reflexión bourdiana sobre el estatuto de las ciencias sociales.

Para comprender la relación de fundamentación entre fenomenología y sociología que se expresa en esta particular apuesta metodológica, comenzaremos describiendo brevemente el tono de la formación de Bourdieu en filosofía; luego expondremos las condiciones sociales de producción que permiten encuadrar su pasaje de la filosofía a la socioantropología hacia finales de la década de 1950 en Argelia, para mostrar finalmente que su propuesta metodológica relativa a superar la oposición entre "explicar y comprender" encuentra un antecedente clave en la enseñanza de Merleau-Ponty, que el aprendiz de sociólogo "manejaba" en el marco de la formación de su babitus filosófico.

\section{De la filosofía a la socioantropología}

Nacido en 1930 en Denguin (Bearn), un pueblo del sur de Francia, e hijo de un cartero de origen campesino, Pierre-Félix Bourdieu cursó su carrera de Filosofía en la École Normale Supérieure (ENS) gracias a una serie de becas por buen rendimiento académico. Se graduó como Agregado en Filosofía en 1954

5 En un sentido estrictamente bourdiano, utilizamos méconnu en este caso, para indicar una negación de saber, fundamentada en el reconocimiento de los linajes legítimos dónde ubicar la trayectoria del autor, fruto de un estado de relación de fuerzas en el campo intelectual.

6 No utilizamos la noción de fundamentación en sentido fenomenológico. 
con una traducción comentada de Animadversiones de Leibniz, en la promoción de Jacques Derrida, Louis Marin y Jean-Claude Passeron. La formación filosófica de Bourdieu se encuentra marcada tanto por los estudios en filosofía de las ciencias, lógica e historia, como por la filosofía fenomenológica que marcó a toda esa camada de normaliens (Pinto, 2007).

Para comenzar, señalamos que para el curso de ingreso de la ENS, Bourdieu lee La estructura del comportamiento de Merleau-Ponty (1942), quien en esos años realizaba en sus clases una interpretación de la obra de Husserl — principalmente de la fenomenología genética- diferente a la de Sartre, centrándose en Ideas I y II (Husserl, 1962, 1997), Experiencia y Juicio (Husserl, 1980) y las Lecciones sobre la fenomenología de la conciencia interna del tiempo (Husserl, 2002). La formación de Bourdieu en fenomenología es fuerte en esos años, también en lo que se refiere a Heidegger y sus estudios sobre el tiempo público y la historia presentes en Ser y Tiempo (Heidegger, 2002), así como las investigaciones realizadas por Husserl en Ideas Iy II (Bourdieu, 1987, 2003) siempre mediadas por la enseñanza merleaupontyana.

En un contexto filosófico marcado por la toma de posición frente al existencialismo sartreano y la incipiente entrada del estructuralismo en ciencias sociales (Bourdieu y Passeron, 1967; Bourdieu, 1987, 2003, 2004); para Bourdieu (1987: 15), Merleau-Ponty ocupaba en el campo académico francés un lugar aparte:

Se interesaba por las ciencias del hombre, por la biología, y daba la idea de lo que puede ser una reflexión sobre el presente inmediato -por ejemplo, con sus textos de historia, sobre el partido comunista, sobre los procesos de Moscú- capaz de escapar a las simplificaciones sectarias de la discusión política. Parecía representar una de las salidas posibles fuera de la filosofía charlatana de la institución escolar.

Las otras salidas, según Bourdieu (1987), la representaban la tradición de la filosofía de las ciencias en las que se encontraban Bachelard, Canguilhem, Vuillemin, y Koyre. Las referencias de Bourdieu a las obras de Bachelard y Canguilhem son bien conocidas, ya que han sido destacadas tanto por el propio sociólogo (Bourdieu, 1987, 2004, entre otros) como por destacados analistas (entre ellos Wacquant, 1992; Baranger, 2004), teniendo gran peso en la configuración bourdiana de la sociología como ciencia, algo que se ve con claridad en los postulados sobre la construcción del objeto de estudio y la vigilancia epistemológica en El oficio de sociólogo (Bourdieu, Chamboredon y Passeron, 1973). ${ }^{7}$ No obstante, es la referencia a Merleau-Ponty la que que-

7 Se puede leer con mucho interés las transformaciones autocríticas, las diferencias y desarrollos epistemológicos posteriores de los dos autores centrales de El oficio... en el caso 
remos destacar de una manera particular, sobre todo teniendo en cuenta que ella opera en dos niveles:

a) Un primer nivel remite a la "antropologización" de problemáticas fenomenológicas (Martínez, 2007; Dukuen, 2013) presente de forma ya temprana en la obra de Bourdieu. En sus primeros artículos de investigación empírica (Bourdieu, 1962, 1963), en los cuales se produce su pasaje de la filosofía a la socioantropología, Bourdieu reasume problemáticas fenomenológicas propias de su formación como filósofo, relativas al cuerpo propio (Leib) y la temporalidad preobjetiva, en especial en la sociedad tradicional campesina kabil y el Bearn francés, cuyo fundamento son las investigaciones de Husserl (2002) y Merleau-Ponty (1945).

Esta reasunción, que hemos analizado extensamente en otros trabajos (Dukuen, 2011b; 2012, 2013) da lugar a la noción de habitus y a la Teoría disposicional de la práctica (Hong Sung-Min, 1999; Martínez, 2007; Grange, 2011; Rey, 2011) que son marca distintiva de la obra bourdiana. Además, en esa línea se comprenden dos cuestiones importantes: tras graduarse en filosofía - y hasta su partida a Argelia en 1955 para realizar el servicio militar-, Bourdieu fue profesor del Liceo de Moulins, donde dedicó el final del curso que dictaba a la enseñanza de la concepción de la temporalidad en Husserl.

Además, en esa misma época, y bajo el influjo claro de la fenomenología merleaupontyana, Bourdieu estudiaba fisiología y medicina psicosomática con Georges Canguilhem, quien dirigiría su proyecto de tesis doctoral nunca concretado sobre "las estructuras temporales de la vida afectiva"; investigación en la que siguió trabajando en Argelia hasta decidir suspenderla (Bourdieu, 1987; Sapiro, 2007a y 2007b) y de la que quedan huellas tanto en el anexo sobre "El descubrimiento de la enfermedad", en El desarraigo (Bourdieu y Sayad, 1964) como en Esbozo de una teoría de la práctica (cfr. Bourdieu, 1972: 253).

b) Otro nivel de referencia es el punto de encuentro entre una serie de reflexiones gnoseológicas de Merleau-Ponty, y ciertos presupuestos epistemológicos y metodológicos de las investigaciones antropológicas de Bourdieu. Un caso ejemplar, en el que nos detendremos aquí — por formar parte del su pasaje de la filosofía a la socioantropología - son los señalamientos metodo-

de Bourdieu en Ciencia de la ciencia y reflexividad (2001), su último curso en el Collège de France; en el caso de Passeron en esa notable obra titulada El razonamiento sociológico (2011). Para un análisis de los puntos de encuentro y desencuentro entre ambos, destacamos los trabajos de Baranger (2004: 59-88, 147-204), Martínez (2007: 272-281) y Moreno Pestaña (2011). 
lógicos realizados en la primera gran investigación empírica que Bourdieu dirigirá: nos referimos a Trabajo y trabajadores en Argelia, publicada en 1963 , y la conexión fuerte y desconocida que presenta, en el marco de la referencia explícita a los "clásicos" de la sociología (Marx, Durkheim y en especial Weber), con la forma en que Merleau-Ponty anteriormente (1945) había descrito ciertos lineamientos del "análisis existencial" del mundo humano y del vínculo entre filosofía y sociología (Merleau-Ponty, 1960).

Los textos no viajan con sus contextos, solía decir Bourdieu (2000). Como su pasaje de la filosofía a la socioantropología se produce durante su estadía en Argelia para realizar el servicio militar, es central comprender ese particular contexto de producción signado por la guerra y la revolución, que encuentra un primer remate con la publicación en 1958 de un Que sais-je? titulado Sociología de Argelia (Bourdieu, 1958). Tomaremos como punto de partida esta obra - basada primeramente en un trabajo sobre fuentes secundarias - para dar cuenta de las condiciones sociales de producción que contribuyen a que Bourdieu dirija las encuestas (entre 1958-1960) que dan lugar a sus primeras investigaciones socioantropológicas. Será en esas investigaciones donde analizaremos la apuesta metodológica relativa a superar la oposición entre "explicar y comprender" propuesta en Trabajo y trabajadores en Argelia (1963), que forman parte de una verdadera experimentación, tanto epistemológica como metodológica, donde se cruzan de forma original filosofía, antropología y sociología.

\section{Condiciones sociales de producción de las investigaciones de Bourdieu en Argelia}

El reciente egresado de Filosofía Pierre Bourdieu llega a Argelia en 1955, siendo destinado allí para hacer su servicio militar (originalmente en Versalles) por mal comportamiento, tras haber tenido una fuerte discusión con oficiales de alto rango que querían convertirlo a la "Argelia francesa" (Bourdieu, 2004: 54). Las consecuencias de este incidente son claves en la trayectoria intelectual y política del autor, y marcan, como él mismo lo ha señalado, "un momento crítico" en la transformación de su visión del mundo (Bourdieu, 2004: 78). Como indica Martín Criado (2006: 15), ${ }^{8}$ allí nacen sus compro-

8 En lo que se refiere a la comprensión de Sociología de Argelia nuestra deuda mayor es con los análisis de Lane (2000) y Martín Criado (2006) que retomamos. Sobre el "periodo argelino" y la antropología de Bourdieu, debemos destacar los trabajos de Addi (2002), Vázquez 
misos políticos públicos con la población argelina - que lo acompañarán hasta su muerte- y se generan sus primeras investigaciones socioantropológicas, siendo la Cabilia la región donde centra sus tres estudios de Etnología (Bourdieu, 1972, 1980; Reed-Danahay, 2006: 69-98) que son el fundamento de la praxeología o Teoría de la práctica, en el marco del paradigma antropológico cabil (Addi, 2002).

En 1956, Bourdieu es asignado a un regimiento de infantería en la región de Cheliff, con la tarea de ayudar a escribir cartas y realizar guardias nocturnas. Al poco tiempo, es trasladado al gabinete militar del Gobierno General en Argel, gracias a la intervención de su madre que se contactó con un coronel de origen bearnes (Bourdieu, 2004: 54-57). En la Biblioteca del Gobierno General, durante 1957, escribe Sociología de Argelia (publicada en 1958), y en el marco de esa tarea conoce a los principales investigadores sobre este país. Entre ellos se destaca el director de la Biblioteca, Emile Dermenghen especialista en el Islam, quien lo contacta con una serie de investigadores como Jacques Berque y André Nouschi (Bourdieu, 2003: 84), historiador y militante comunista de quien Bourdieu obtiene ayuda para escribir su libro (Martín Criado, 2006: 37-38; Nouschi, 2003).

En Sociologia de Argelia (1958), Bourdieu retoma las investigaciones sociológicas y antropológicas producidas durante el periodo colonial, poniendo el eje en el análisis de tres "culturas" bereberes: cabilios, chaouias y mozabitas, y la población arabófona, considerando luego los elementos comunes y analizando la desestructuración que la situación colonial ha ejercido sobre esas poblaciones. La investigación está basada especialmente en una detallada investigación bibliográfica y en conocimientos de primera mano, bajo el objetivo de "decirles a los franceses, sobre todo de izquierda, lo que ocurría realmente en un país del que la mayoría ignoraba casi todo, y ello, una vez más, para ser útil en algo, y quizás también para conjurar la mala conciencia de testigo impotente de una guerra atroz" (Bourdieu, 2004: 56-57).

Desde la perspectiva de Lane (2000: 12-13), Bourdieu escribe su libro en discusión frontal con la posición de otros investigadores contemporáneos, en especial Germaine Tillion, etnógrafa que habiendo sido consejera del gobernador general de Argelia entre 1955-1956, sostenía una posición reformista basada en el subdesarrollo de las masas argelinas. En su libro de 1957, titulado $L^{\prime}$ Algérie, se sostenía que la introducción de medicinas europeas, el alivio del hambre y la economía monetaria habían minado la estabilidad de la sociedad

García (2002), Reed-Danahay (2006), y los números 21 y 27-28 de la revista Awall (AAvv, 2000, 2003). 
tradicional, sin integrarla en una economía racional moderna. Y proponía una política ilustrada de ayuda y educación para producir una "verdadera mutación social” de la tradición hacia la modernidad, que produjera la pacificación y la desaparición de las quejas del nacionalismo argelino.

La posición de Tillion jugaba un papel fundamental en el humanismo reformista de izquierda, y en los debates del momento. Bourdieu, por el contrario, va a insistir sobre la integración y funcionalidad de las culturas originales y señalar que el reformismo es totalmente irrealista: la lógica objetiva del sistema colonial basada en la búsqueda de la explotación económica implica necesariamente la desestructuración de las culturas argelinas, y su "estado de subdesarrollo". Eso explica que las reformas propuestas por Tillion no se realizaron a lo largo de un siglo. Así, Bourdieu tomaba partido por la causa independentista, lo cual hizo que Sociología de Argelia fuera muy mal recibida por los defensores de la Argelia francesa, y nuestro autor pasase a formar parte de las listas negras de la ultraderecha colonialista.

Nacido "de un impulso cívico más que político" (Bourdieu, 2003: 85), Sociología de Argelia es el libro de Bourdieu con más reediciones corregidas (ocho en total), y en ese periplo se puede ver la radicalización de la postura de Bourdieu, llegando a defender por única vez una salida revolucionaria; así como un claro cambio en la comprensión del acontecimiento que se ve expresado en el uso del lenguaje (Lane, 2000: 17). En 1960, Bourdieu publica "Guerra y transformación social en Argelia”, lo que en 1958 era señalado como un "conflicto" (Bourdieu, 1958: 125), aquí es analizado como una "guerra", dando cuenta de las acciones del brazo armado del Frente de Liberación Nacional.

En el artículo siguiente, de 1961, titulado "Revolución en la revolución”, Bourdieu analiza las posibilidades de que la guerra de liberación se transforme en una revolución socialista. La segunda edición, de 1961, implica cambios importantes relativos a las referencias teóricas — sustituciones y desaparición de marcos teóricos- fruto del afinamiento de ciertas lecturas al comienzo "escolares" - en especial Weber y Levi-Strauss - que producen variaciones en el análisis de los distintos sistemas o sociedades - "culturas", en la edición de 1958 - gracias a los resultados obtenidos de las investigaciones llevadas a cabo en encuestas, una en 1958 y dos en 1960 (Sapiro, 2007a: 44) y a las conclusiones alcanzadas en los dos artículos que hemos referido.

En ese sentido se puede comprender que Bourdieu sostenga la propuesta de que el "sistema colonial" sea "radicalmente destruido [...] de arriba a abajo" por la población argelina, para que asuma "su propio destino en completa 
libertad y responsabilidad", y construyan una sociedad nueva, producto de la "participación activa, creativa y resuelta en una tarea común" (Bourdieu, 1961: 125-126, citado en Lane, 2000: 18).

En 1962 se produce la traducción en inglés de Sociología de Argelia, titulada "The Algerians", que concluye con una versión extendida de "Revolución en la revolución". La tercera edición de 1963 es casi idéntica a la segunda, salvo por la sección final donde se expresa un posicionamiento contundente frente al desarrollo político. Allí Bourdieu sostiene por única vez una salida revolucionaria: la sociedad argelina, "profundamente revolucionada por la colonización y la guerra" demanda "objetivamente, soluciones revolucionarias". Al obtener Argelia su independencia en 1962, el éxodo masivo de europeos hace "del acceso a la independencia la oportunidad para una revolución económica y social", lo cual implica elegir "entre el caos, o una forma de socialismo que se corresponda con la realidad" (Bourdieu, 1963: 126, citado en Lane, 2000: 18).

Esta sección será reescrita en 1985 (Lane, 2000: 18-20) a la luz de una ida y vuelta entre acontecimientos e investigación: en sus investigaciones tituladas Trabajo y trabajadores en Argelia (1963) y El desarraigo (1964), Bourdieu va tomando distancia de sus tomas de posición más radicales. Como señala Lane (2000: 19), en los artículos escritos entre 1962-1963 enfrenta la posición de Fanon y de Sartre, cargada, según Bourdieu, de un voluntarismo político totalmente irrealista. Allí ya se puede ver un primer desacuerdo tanto teórico como político con la fenomenología existencialista de Sartre, y en especial con el modelo de sujeto revolucionario propuesto en Questions de méthode (Sartre, 1960).

Así Bourdieu se vuelve cada vez más escéptico al observar el desarrollo de los acontecimientos posindependencia, llamando la atención sobre el error de creer que los campesinos y subproletarios son una fuerza revolucionaria per se, ideología propagada por la burguesía y la burocracia de Estado para asegurarse una base de apoyo entre ellos (Bourdieu, 1985: 125, en Lane, 2000).

Sin embargo, la investigación originaria que da como resultado Sociologia de Argelia de 1958 - hecha la salvedad de las variaciones en las ediciones posteriores - no está basada en trabajos de campo/encuestas realizadas por el propio Bourdieu. Egresado de Filosofía, no se encontraba ni teórica ni metodológicamente preparado para desplazarse como investigador a la antropología y a la sociología, disciplinas consideradas "menores" para un graduado de la ENS. Justamente para comprender ese pasaje y el peso específico del habitus 
filosófico en esta experimentación teórico/metodológica de “tránsfuga disciplinar", vamos a detenernos a continuación en las investigaciones empíricas realizadas por Bourdieu en Argelia a partir de 1958, y muy especialmente en la obra colectiva Trabajo y trabajadores en Argelia, donde "en la marcha" y sobre el terreno, comienza a aprehender el "oficio de sociólogo”.

\section{Aprehender el oficio en el terreno}

A partir de 1958 — tras finalizar su servicio militar-, Bourdieu dictó cursos en la Universidad de Argel sobre Kant, Durkheim, Saussure y Levi-Strauss. El periodo como docente en esa casa de estudios le permite interiorizarse más en la variante estructuralista de las ciencias humanas y reclutar estudiantes para realizar las investigaciones que darán como resultado dos obras en colaboración: Trabajo y trabajadores en Argelia (1963) y El desarraigo (1964), y artículos que resumen dimensiones de esas investigaciones, como "El temor al desempleo en los obreros argelinos" (Bourdieu, 1962b) y "La sociedad tradicional” (Bourdieu, 1963). Además, Bourdieu hace trabajo de campo en su pueblo natal, en la zona del Bearne francés, entre 1959 y 1960, lo que da como resultado un largo artículo: "Celibato y condición campesina", publicado en 1962 en la revista Estudios Rurales.

En este trabajo fundamental, Bourdieu introduce por primera vez la noción de habitus retomando el artículo seminal de Mauss ([1936]1979) sobre "técnicas y movimientos corporales"; y centrándose en la relación entre los sexos en la sociedad campesina, se pueden encontrar las huellas de la génesis de una teoría de la violencia simbólica corporal de orientación fenomenológica; cuestiones de las que nos hemos ocupado en otra parte (Dukuen 2011; 2013). Las investigaciones argelinas que dan como resultado los libros Trabajo y trabajadores en Argelia y El desarraigo, tienen su fundamento en tres trabajos de campo/encuestas: uno en 1958 y dos en 1960 (Sapiro, 2007a: 44). Bourdieu emprende esta tarea con una selección de estudiantes entre los que se encuentra Abdelmalek Sayad — coautor de El desarraigo- y jóvenes estadísticos del Institut National de la Statistique et des Études Économiques (INSEE), que marchan hacia Argelia para formar el Servicio de Estadísticas a pedido del presidente De Gaulle. Entre ellos están Alain Darbel, Jean Paul Rivet y Claude Seibel, con quien Bourdieu escribirá Trabajo y trabajadores en Argelia y formarán, junto a una serie de investigadores argelinos, la Association pour la Recherche Démographique, Économique et Sociale (ARDES). 
Martín Criado (2006: 54-55) señala que las investigaciones empíricas que dieron lugar a estos dos libros tienen como punto de partida un encargo del ejército francés: estudiar las poblaciones desplazadas tras las denuncias en la prensa. A través de una serie de decretos, a partir 1956 Argelia fue dividida en tres zonas: "de pacificación", de "operación militar", y "prohibidas" para la población. En la "Introducción” a El desarraigo, Bourdieu y Sayad (1964: 11) señalan que:

entre 1954 y 1957 muchos campesinos fueron pura y simplemente expulsados de sus pueblos (...) solamente a partir de 1957, y en ciertas regiones (...) la política de reagrupamiento adquirió un carácter metódico y sistemático. Según las instrucciones oficiales, el objetivo primordial de las zonas prohibidas era "evacuar un país incontrolado y sustraer la población a la influencia rebelde".

Ante esta situación, Bourdieu es llamado a dirigir las investigaciones de campo e interpretar los datos estadísticos. Siendo su primera investigación empírica importante y contando especialmente con una formación filosófica, Bourdieu opera una redefinición del objeto de estudio como una negativa a realizar lo que podríamos calificar como "un trabajo de inteligencia": la demanda del Estado colonizador es invertida, de tal forma de estudiar la desestructuración producida por la colonización sobre la población argelina (relativa a la relación con el trabajo y el hábitat en subproletarios y campesinos), en el pasaje de una sociedad precapitalista a una capitalista en el contexto de guerra y desplazamientos-reagrupamientos forzados.

El trabajo de campo se centra tanto en los "campos de reagrupamientos" como en las ciudades, y combina las más variadas técnicas socioantropológicas: fotografías, observación participante, grabaciones clandestinas de conversaciones, entrevistas en profundidad, reconstrucciones de sistemas de parentesco, descripción de rituales, entre otros (Martín Criado, 2006: 5556). Cabe subrayar la gran peligrosidad en la que se desarrolló esta investigación, cuestión que Bourdieu recuerda en su "autoanálisis" (Bourdieu, 2004: 65-73).

En este contexto de guerra, aparecen claramente las primeras preocupaciones bourdianas de orden epistemológico y metodológico que darán lugar en 1968 a la escritura de El oficio de sociólogo y que tiene un claro antecedente en la "Introducción" de Trabajo y trabajadores en Argelia. Los investigadores eran seguidos por los militares, y en ese marco las entrevistas podían derivar en respuestas "correctas". De allí las primeras preocupaciones por la "vigilancia epistemológica" que implicaban en la práctica poder establecer una comunicación no violenta con los campesinos, en un contexto donde la violencia es lo que primaba. 
En 1960, como señalamos con anterioridad, Bourdieu se ve obligado a abandonar Argelia por estar marcado en una lista negra de la ultraderecha. Gracias a una colega de la Facultad de Argel, que había sido compañera de Raymond Aron en la École Normale Supérieure, Bourdieu entra en relación con él. Ante el peligro que corre su vida en Argelia, Aron le ofrece ser su ayudante en la Sorbona a donde Bourdieu se dirige, para luego trasladarse a Lille donde enseña entre 1962 y 1963.

Bajo la mira del sociólogo — quien iba ser su director en una tesis basada en las investigaciones argelinas, pero que no consideró digna de Bourdieuparticipa de la escritura en colaboración de Trabajo y trabajadores... y El desarraigo. Allí ya se puede ver la importancia creciente que Weber toma en su obra, algo que no es extraño si se tiene en cuenta que Aron era un reconocido especialista en el sociólogo alemán (y quien introdujo su obra en Francia) y que inició a Bourdieu en la lectura de Schutz (Bourdieu, 2004: 80), cuyo primer libro aborda inicialmente una reelaboración, fenomenológicamente fundamentada, de la Teoría de la acción de Weber (Schutz, 1993). Sin embargo, contra lo que se podría suponer, Bourdieu llega primero a Weber por otra vía, que debemos destacar y se hace comprensible si no perdemos de vista su formación en filosofía fenomenológica: su primer contacto con el autor fue gracias a la lectura de Las aventuras de la dialéctica de Merleau-Ponty, como lo aclara en una entrevista realizada por Schultheis y Pfeuffer:

Fue a través de Las aventuras de la dialéctica de Merleau-Ponty, que encontré realmente impresionante. Ese libro tuvo un fuerte impacto en mí, en mi juventud, y recuerdo que existía un breve capítulo en el cual él hablaba de Weber. Creo que esa fue la primera vez que oí hablar sobre Weber (Bourdieu, Schultheis y Pfeuffer, 2011: 112).

Bourdieu se refiere sin dudas al primer capítulo de Las aventuras de la dialéctica, titulado "La crisis del entendimiento", donde Merleau-Ponty (1955) retoma in extenso a Weber, lo cual se comprende en el marco de su especial interés por "las ciencias del hombre" (Merleau-Ponty, 1977). Cabe destacar que en el contexto de su colaboración con Aron, Bourdieu produce el desplazamiento de una lectura propiamente escolar de Weber - como la producida de Sociología de Argelia - a una profundización productiva en lo que concierne a:

a) Los estudios sobre el ethos protestante y los orígenes del capitalismo (Weber, 2007) que le servirá como modelo teórico para analizar las transformaciones de la sociedad argelina y en especial lo que podríamos llamar "la estructura del comportamiento" de trabajadores, subproletarios y campesinos en su vínculo con la temporalidad y el trabajo (Martínez, 2007: 31-58). 
b) La relectura de la noción de clase social mediante el cruce entre la tradición marxista y la weberiana que se esboza en Trabajo y trabajadores... y se cristaliza en el artículo de 1966 Condición de clase y posición de clase (Bourdieu, 1966).

c) La impronta metodológica tanto en la construcción de tipos ideales (Weber, 2007) — sea el caso de la noción de "sociedad tradicional" (Bourdieu, 1963) - como en la interpretación de la relación entre explicar y comprender en el encuentro entre estadística y sociología. En esta relación nos detendremos, porque encuentra un antecedente clave y desconocido en una serie de señalamientos de Merleau-Ponty (1945), revisitando primeramente la apuesta metodológica de Bourdieu en Trabajo y trabajadores en Argelia.

\section{Explicar y comprender o una apuesta metodológica de impronta merleaupontyana}

Trabajo y trabajadores en Argelia ${ }^{9}$ es la primera obra colectiva en la que participa Bourdieu, resultado de "una colaboración bidisciplinar entre sociología y estadística, y acorde con la utilización concertada de métodos diferentes, aunque complementarios" (Bourdieu et al., 1963: 12), basada en los trabajos de campo/encuestas que estuvimos señalando con anterioridad. Esta obra presenta dos partes muy bien definidas. Una primera en la que Darbel, Rivet y Seibel efectúan el análisis estadístico de la sociedad argelina, donde se definen las principales estructuras de la población de las ciudades y de los campos de reagrupamiento forzoso. La segunda parte, titulada "Estudio sociológico" y escrita en exclusividad por Bourdieu, se basa en entrevistas realizadas a una submuestra al 10\%, con el propósito de "determinar las actitudes y las opiniones de los individuos interrogados" (Bourdieu et al., 1963: 13).

Esta división tajante del trabajo sociológico puede llamar la atención, teniendo en cuenta que Bourdieu la criticará in extenso unos años después en El oficio de sociólogo (Bourdieu et al., 1973) y no la retomará en ninguna de sus obras posteriores. Sin embargo, ya en Trabajo y trabajadores en Argelia Bourdieu comienza a romper con ella, lo cual contra una lectura profética de las trayectorias intelectuales evidencia un gran trabajo de objetivación de las propias operaciones de construcción del objeto de estudio. Algunos señalamientos importantes de esta operación crítica se verán ya en la Introducción

9 Trabajo y trabajadores en Argelia nunca fue reeditada en francés ni traducida, sólo existe una versión abreviada y corregida que publicó Bourdieu en 1977 bajo el título de Argelia 60 (Bourdieu, 2006). 
de la obra, escrita por Bourdieu bajo el título de "Estadística y sociología". Aquí podremos observar una primera apuesta metodológica de Bourdieu, en la cual defiende la complementariedad entre métodos cuantitativos y cualitativos, llamando la atención sobre que "lo que hay que denunciar no es el uso de la estadística, sino el fetichismo de la estadística" (Bourdieu et al., 1963: 9), porque "en la dialéctica entre la hipótesis y la verificación estadística, la oposición clásica entre la explicación y la comprensión es superada (Bourdieu et al., 1963: 10-11).

Detengamos un instante en este punto: Bourdieu alude a la superación ${ }^{10}$ de la oposición excluyente señalada por Dilthey (1900), contra el positivismo, entre explicar como método propio de las "ciencias de la naturaleza" (Naturwissenschaften) y comprender como método propio de las "ciencias del espíritu" (Geisteswissenschaften). Como explica Ricoeur (2001: 131 y ss), en Dilthey la alternativa excluyente indica que o bien se explica, a la manera del científico naturalista; o bien se comprende, como hace el historiador cuando interpreta, como provincia particular de la comprensión.

Dilthey está diferenciando dos esferas: la región de la naturaleza sometida a la matematización desde Galileo y desde Stuart Mill a la lógica inductiva; de la región del espíritu, propia de las individualidades psíquicas, hacia cuyo interior cada psiquismo puede trasladarse, dado que se da en signos exteriores: "llamamos comprensión al proceso por el cual conocemos algo psíquico con la ayuda de signos sensibles que son su manifestación" (Dilthey citado por Ricoeur, 2001: 132). Entre esos signos están aquellas manifestaciones fijadas durablemente, conservadas especialmente por la escritura. La interpretación es el arte de comprender esos signos particulares.

Contra la oposición entre explicar y comprender, que permite preguntarse cómo fundamentar el carácter científico de una sociología que pretende ir más allá del ensayismo, recurriendo a las posibilidades de la estadística sin caer en el positivismo ramplón ni en el psicologismo, Bourdieu se posiciona a favor de la legitimidad de las ciencias sociales en cuanto ciencias: por un lado, contra el modelo rey de las ciencias naturales y exactas - donde no encontramos la pregunta por el sentido-; por otro, contra la derivación de la sociología positivista en el "empirismo abstracto” (Wright Mills, 1961; Bourdieu et al., 1973); y al mismo tiempo, contra la "gran teoría” al estilo Parsons. Ni

10 Martín Criado (2006: 84) señala que "la superación” era una forma retórica muy común entre los "maestros" intelectuales de Bourdieu —es el caso de Merleau-Ponty (1945) con la superación del empirismo e intelectualismo-, que consiste en oponer explicaciones contrarias y proponer una tesis que exprese el carácter parcial de ambas, y las supere. 
teoricista ni empirista, la sociología se construye como investigación empírica teóricamente fundada.

Denis Baranger ha señalado que con esta posición epistemológica, relativa a sobrepasar la oposición entre explicar y comprender, Bourdieu "busca superar la dualidad entre la formación científica y la humanística sin caer sin embargo en el positivismo" (Baranger, 2004: 92). Esto indica una reasunción del viejo debate — cuyo epígono es Durkheim (2008) — con la filosofía como reina madre del saber sobre el mundo humano, y una salida posible desde las ciencias sociales frente a la crítica al positivismo y a la actitud objetivista propuesta por Husserl (2010) en La crisis de las ciencias europeas y la filosofía trascendental.

En ese sentido, el novel sociólogo comienza a realizar un ajuste de cuentas (que nunca cesará, como se ve en Meditaciones pascalianas de 1997) con las tensiones y desgarros que producen las disposiciones contradictorias que lo habitan, propias de su formación de origen como filósofo y el hecho de aceptar que se está transformado — y rebajado en la jerarquía intelectual francesa-en y por la práctica, en un antropólogo/sociólogo.

Dicho esto, la superación de la oposición entre explicar y comprender que Bourdieu presenta como su apuesta inicial en términos metodológicos, se puede ordenar en un triple movimiento, desplegado a partir del análisis hecho por Baranger (2004: 92 y ss), a lo que sumaremos el punto de encuentro originario y original con las tesis de Merleau-Ponty sobre el mismo tópico. El triple movimiento es el siguiente:

a) En torno a la explicación, desde un punto de vista "durkheimiano".

b) En torno a la comprensión/interpretación, desde el punto de vista weberiano/merleaupontyano.

c) En torno al método para una "descripción de las clases sociales”, desde el punto de vista de Marx.

Teniendo en cuenta el objeto de este artículo, si bien describiremos los tres puntos, nos detendremos especialmente en el $b$; invitando al lector a profundizar en $a$ y $c$ en el notable trabajo de Baranger (2004: 89-94, 113-117) sobre epistemología y metodología en la obra de Bourdieu. Dicho esto, comencemos por el primer punto:

a) En torno a la explicación, para Bourdieu el dato estadístico permite establecer relaciones entre variables. "Desde este punto de vista 'durkheimiano’ (...) el análisis de variables está planteado entonces como el sustituto de la experimentación para la investigación sociológica” (Baranger, 2004: 92), ya que permite no sólo establecer nuevas relaciones y por ello producir nuevas 
hipótesis, sino también poner en tela de juicio aquellas que han guiado la investigación:

Además de su valor probatorio, la estadística tiene un valor heurístico puesto que permite descubrir relaciones en las que no se había pensado (...) es para el sociólogo lo que la experiencia es para el físico; la estadística opone a la hipótesis la resistencia de lo dado y por esta vía obliga a forjar nuevas hipótesis (Bourdieu et al., 1963: 10).

Llamamos la atención sobre el índice realista relativo a la "resistencia de lo dado", que el propio Bourdieu corregirá críticamente en El oficio de sociólogo (Bourdieu et al., 1973) entendiendo el "dato" estadístico como una construcción. Esto muestra, nuevamente, el carácter lento y trabajoso que implica el proceso de ruptura múltiple mediante el cual Bourdieu fue produciendo los supuestos fundamentales de su teoría.

b) Tras fundamentar la importancia de la estadística para la sociología como ciencia a partir de un enfoque "durkheimiano", en el texto "enseguida aflora la orientación weberiana” (Baranger, 2004: 92):

El método estadístico permite captar las cadenas causales en las que están insertas, gracias al uso de hipótesis interpretativas, motivaciones objetivamente dotadas de sentido. $\mathrm{E}$, inversamente, los datos estadísticos, en la medida en que están referidos al desarrollo o a las consecuencias de una actitud que encierre en sí algo comprensible, sólo son "explicados" verdaderamente si se los interpreta realmente de modo tal que alcancen a revestir un sentido en el caso concreto. Por lo tanto, la interpretación causal de un comportamiento o de una opinión sólo se logra cuando la acción manifiesta y sus motivaciones han sido aprehendidas y el vínculo que las une se ha vuelto comprensible bajo el aspecto del sentido. Así, las regularidades estadísticas tienen un valor sociológico solamente en el caso de que puedan ser comprendidas. E inversamente las relaciones subjetivamente comprensibles sólo constituyen modelos sociológicos de los procesos reales si se las puede observar con un grado de confianza significativo (Bourdieu et al., 1963: 11).

En este párrafo se puede observar una suerte de dialéctica donde el método estadístico permite captar cadenas causales sólo mediante hipótesis interpretativas que las hagan comprensibles - lo reconozca o no el sociólogo, no hay dato puro, dirá Bourdieu contra el positivismo en El oficio de sociólogo (Bourdieu et al., 1973). Así, la acción y sus motivaciones — se reconocerá el lenguaje weberiano - objetivadas bajo la forma estadística, cobran valor sociológico plenamente cuando "revisten un sentido en el caso concreto", cuando son "comprensibles bajo el aspecto del sentido".

Se observa que frente a Dilthey, la comprensión no supone aquí la referencia a un psiquismo interior como fuente de la acción, sino que el punto de partida es el propio comportamiento/acción como observable sociológico 
primario. De alguna manera ésta es la reacción bourdiana (de orientación durkheimiana, si dudas) contra una posible deriva psicologista que el autor observará incluso en Weber (cfr. Bourdieu, 1971 a y b). Es en la dialéctica entre método estadístico e hipótesis interpretativas donde se puede observar el germen de un intento - no formulado explícitamente todavía- de una superación entre objetivismo y subjetivismo (Bourdieu, 1972, 1980), centrado en la imbricación entre explicación y comprensión.

Al leer la argumentación, se evidencia que la tesis de Bourdieu conecta con la posición weberiana sobre "el sentido de la acción", aspecto sobre el cual ha llamado la atención Baranger (2004: 92 y ss) de forma contundente. Pero si no perdemos de vista que Bourdieu lee a Weber por primera vez mediado por Merleau-Ponty (y luego por Aron), vale detenerse en la impronta del fenomenólogo en las propias argumentaciones de Bourdieu (a primera vista sólo weberianas) de la que encontramos huellas en la tesis de Trabajo y trabajadores en Argelia, sin perder de vista que la ausencia de citas no debe llevar a engaños: teniendo en cuenta que en ese contexto la formación de Bourdieu en fenomenología es fuerte y reciente, que la importancia de Merleau-Ponty en esa formación es central y que la lectura de Weber esté mediada por su obra, cabe destacar su encuentro fuerte con algunos señalamientos puntuales del fenomenólogo francés, previos a Las aventuras de la dialéctica, presentes de hecho en su gran obra Fenomenología de la percepción (1945), que Bourdieu conocía in extenso junto con La estructura del comportamiento (1942) (Sapiro, 2007a, 2007b).

Para comenzar podemos señalar una coincidencia muy clara de la tesis de Bourdieu con lo señalado por Merleau-Ponty 18 años antes en el apartado de la Fenomenología de la percepción, titulado "La espacialidad del cuerpo propio y la motricidad". ${ }^{11}$ Allí, contra la fisiología empirista, que recurre a la explicación causal de ciertos "trastornos psíquicos" — como la afasia, el miembro fantasma, la anosognosia, la ceguera psíquica - y contra la psiquiatría intelectualista que frente a ellos apela a la reflexión, Merleau-Ponty afirma que "no hay explicaciones sin comprensión" (Merleau-Ponty, 1945: 134) y propone "un nuevo modo de análisis — el análisis existencial- que supera las alternativas clásicas del empirismo y del intelectualismo, de la explicación y de la reflexión" (Merleau-Ponty, 1945: 158).

11 Se puede leer sobre este punto la investigación de García (2012) y especialmente el artículo de Pintos Peñaranda (2008), que aborda la relación crítica y productiva de Merleau-Ponty con la obra del psiquiatra Goldstein y el "análisis existencial” del célebre "caso Schneider", que sirve como fuente empírica del debate. 
Bien podemos preguntarnos qué significa esta propuesta en la obra de ese particular fenomenólogo, sin dudas el más interesado en las ciencias del hombre. Para entenderlo en el marco general del encuentro entre filosofía y sociología que propone, es pertinente remitirnos a un comentario esclarecedor realizado por Merleau-Ponty en el capítulo "La libertad" de la Fenomenología de la percepción (1945) y a la tesis extensiva propuesta en los artículos "De Mauss a Claude Lévi-Strauss" y "El filósofo y la sociología”, publicados en Signos (1960).

En "La libertad", en el contexto de una discusión con Sartre que recuerda en muchos puntos a la crítica realizada posteriormente por Bourdieu (1972, 1980) en Esbozo... y El sentido práctico, Merleau-Ponty señala que es necesario comprender que "la generalidad y la probabilidad no son ficciones sino fenómenos, y por lo tanto debemos encontrar para el pensamiento estadístico un fundamento fenomenológico" (Merleau-Ponty, 1945: 505).

Ante esta afirmación imperativa nos preguntamos: ¿No aparece allí, delineándose, la pregunta por "el sentido" del dato estadístico que luego preocupará incansablemente a Bourdieu? Podemos arriesgar que, en continuación con esta interpelación de Merleau-Ponty, asoma la propuesta de Bourdieu, cuando afirma -como vimos anteriormente- que "los datos estadísticos (...) sólo son 'explicados' verdaderamente si se los interpreta realmente de modo tal que alcancen a revestir un sentido en el caso concreto" (Bourdieu et al., 1963: 11).

A nuestro entender, la confluencia de perspectivas es notable, y más bien, para ser directos, las reflexiones de Merleau-Ponty operan como uno de los fundamentos disposicionales que conforman el habitus savant _ sin que ello implique una consciencia explícita, obviamente- que permite el pasaje de Bourdieu hacia la antropología: Bourdieu reelabora filosóficamente su relación con la metodología de las ciencias sociales mediante la pregunta estrictamente fenomenológica por el sentido. Entonces habrá que encontrar la generalidad y la probabilidad, de la que hablaba Merleau-Ponty, en el caso concreto, por ejemplo, el del tránsito de obrero a revolucionario presentado en el apartado "La libertad" de la Fenomenología de la percepción: si la generalidad y la probabilidad son fenómenos, se deberá realizar un "análisis existencial" de la economía y la sociedad en la que "existo como obrero", "tal como las llevo en mí" y no como "sistema de fuerzas impersonales" (Merleau-Ponty, 1945: 506).

Tal vez la mejor transposición de este problema a la socioantropología sean justamente los análisis de Bourdieu que van desde las investigaciones 
argelinas sobre la conciencia perceptiva del tiempo en campesinos y subproletarios (1962), hasta el sentido del honor en la sociedad cabil, la casa, su relación quiasmática con el mundo social y su vínculo estructural con la dominación masculina (Bourdieu, 1962a, 1962b, 1972, 1980, 1990, 1998, 2003); cuestiones de las que nos hemos ocupado en otro lado (Dukuen, 2012, 2013; Ralon de Walton y Dukuen, 2013).

Volviendo a Merleau-Ponty, y en continuidad con los desarrollos de la Fenomenología de la percepción, en los artículos titulados sugerentemente "De Mauss a Levi-Strauss" y "El filósofo y la sociología” publicados en Signos (1960), se propone la crítica a la sociología de tipo "objetivista”. Martínez (2007: 151-152) ha señalado que el principal desacuerdo entre las perspectivas de Bourdieu y Merleau-Ponty es la divisoria de tareas que el fenomenólogo propone entre la sociología que describe cosas, y la filosofía que las interpreta ocupándose de la significación: "El sociólogo hace filosofía, en la medida en que se encarga, no sólo de anotar los hechos, sino de comprenderlos. En el momento de la interpretación, es ya filósofo" (Merleau-Ponty, 1960: 127).

Tal vez el problema aquí resida en que Merleau-Ponty apunta contra la sociología y/o antropología encuadrada dentro de la actitud objetivista, siendo las corrientes dominantes en ese entonces: en un extremo, el "empirismo abstracto" de Lazarsfeld, y en el otro, la antropología estructural de Lévi-Strauss. Sin embargo, como señala Graciela Ralon de Walton (2008), el objetivo de Merleau-Ponty, en la propuesta de un diálogo entre fenomenología y ciencias humanas (Merleau-Ponty [1953] 1977), es encontrar un medio común o tercera dimensión entre el punto de vista objetivo (característico de la ciencia) que ve al hombre en tercera persona "sólo a partir de su dependencia respecto del medio psíquico, orgánico y social hasta el punto que su aparición como un 'producto del medio' otorga preeminencia únicamente a las condiciones externas" y el punto de vista subjetivo-reflexivo (proveniente de la filosofía) donde "el hombre es considerado como la fuente absoluta del sentido, otorgándole un primado absoluto a la interioridad, es decir, a la conciencia entendida como una actividad que coloca con plena autonomía objetos inmanentes y transparentes" (Ralon de Walton, 2008: 1).

Para el fenomenólogo francés, esas posiciones extremas impedirían una posibilidad de diálogo entre ciencias humanas y filosofía. En la propuesta de Merleau-Ponty, se hace necesario dilucidar un "intermundo" que en Las aventuras de la dialéctica (Merleau-Ponty, 1955) llama "verdad o historia a hacer", que permite sobrepasar la oposición entre objetivo y subjetivo, pasividad y actividad, autonomía e independencia: en ese "intermundo" los hechos 
son retomados y vividos por un sujeto encarnado y situado socialmente, y, en esa reasunción radica la posibilidad de la comprensión.

Justamente Bourdieu trata tempranamente de encontrar una salida frente al objetivismo y al subjetivismo, y propone, mediante su apuesta metodológica sobre la imbricación entre explicación y comprensión, una superación de esa división. Por ello se puede encontrar una confluencia de los señalamientos merleaupontyanos con las críticas al objetivismo presentes ya en Esbozo de una teoría de la práctica (Bourdieu, 1972).

En ese sentido, entendemos que en este fundamento fenomenológico se asentarán las nociones de ethos en Trabajo y trabajadores... y de habitus en El desarraigo, tal como las retoma Bourdieu de la filosofía aristotélico-tomista mediante el prisma weberiano (Martínez, 2007: 31-59) para comprender la "sociedad tradicional". Ambas nociones buscan dar cuenta justamente de cómo "esas fuerzas impersonales" objetivadas por el analista se encarnan en los agentes mediante una "conciencia perceptiva" del tiempo que diferencia justamente el ethos tradicional del ethos capitalista: la generalidad y la probabilidad aparecen en el caso concreto, tal como lo hubiese querido MerleauPonty.

La clave se encuentra, finalmente, en construir un "hecho social total" (en el sentido de Mauss) en donde lejos de impugnar la investigación antropológica por el origen nacional del investigador (en este caso el país colonialista), ella implique justamente un análisis de la relación entre "sistema colonial" y "situación existencial del colonizado". Ello implica un movimiento productivo entre la reconstrucción de relaciones objetivas "necesarias e independientes" - a la manera de Marx- (que permiten explicar) y la retoma tanto weberiana como merleaupontyana de la experiencia significativa subjetiva (que permite comprender):

No hay conducta, actitud o ideología alguna que pueda ser descrita, comprendida o explicada objetivamente al margen de toda referencia a la situación existencial del colonizado, tal como es determinada por la acción de las fuerzas económicas y sociales características del sistema colonial (....) por el sistema de "relaciones determinadas, necesarias e independientes de las voluntades individuales" por referencia al cual se organizan las actitudes y las conductas. Tal es la responsabilidad real del etnólogo (...) restituir a otros hombres el sentido de sus comportamientos (Bourdieu et al., 1963: 258-259).

En ese sentido, retomando el análisis de Baranger (2004: 92-93) se observa la cristalización de la dialéctica propuesta por Bourdieu entre hipótesis y verificación; guiada por una suerte de juego de "oposiciones complemen- 
tarias" entre Durkheim, Weber y Marx como se señalará tiempo después en la Proposición 0 escolio 2 de La reproducción (Bourdieu y Passeron, 1970: 18-19).

c) Las indicaciones metodológicas y epistemológicas del Prólogo de Trabajo y trabajadores... se coronan con una referencia a la Introducción a la Contribución a una critica de la Economía politica de Marx: "Sin duda el recorte estadístico despedaza la totalidad concreta captada por la intuición, pero solamente para reconstituir nuevas totalidades no menos concretas" (Bourdieu et al., 1963: 11-12). La referencia a Marx (Wacqüant, 2001; Hong Sung-Min, 1999; Gutiérrez, 2003; Karsenti, 2011) que era casi inexistente en Sociología de Argelia, cobra otro peso aquí, ya que se propone un "esbozo para una descripción de las clases sociales” definidas según una serie de criterios pertinentes que permiten reconocer cuatro categorías: sub-proletarios; proletarios; artesanos y comerciantes; y una burguesia moderna (Baranger, 2004: 114-115). El análisis de las clases sociales se realiza poniendo en la mira las posibilidades reales de un proceso revolucionario socialista, que vaya más allá de la propuesta de Sartre/Fanon a las cuales Bourdieu se opone, como señalamos antes. Pero ésa es otra historia.

\section{Conclusiones. Bourdieu, las ciencias sociales y la fenomenología}

El recorrido que hemos realizado por el debut de Bourdieu en su pasaje de la filosofía a la socioantropología, muestra que la tesis presente en el apartado "Comprender" de La miseria del mundo - publicada en 1993- que afirma "contra la vieja distinción diltheyana, hay que plantear que comprender y explicar son una misma cosa" (Bourdieu, 1993: 910), lejos de ser una acomodamiento de última hora frente a las corrientes hermenéutico-interpretativas y "posmodernas", ha sido defendida por Bourdieu desde su primer gran obra socioantropológica de investigación empírica, Trabajo y trabajadores en Argelia de 1963.

Es allí, en un contexto donde la realización del trabajo de campo, de las encuestas y entrevistas presenta una enorme dificultad por la situación de guerra, de reagrupamientos forzados y vigilancia militar, donde Bourdieu ve la necesidad de "comprender" - contra todos los males de este mundo- o sea, de poder ir más allá de lo que las herramientas metodológicas de la sociología empirista pueden brindar, y a partir de un diálogo subterráneo con la fenomenología merleaupontyana poner a trabajar el "dato estadístico", en el marco de las hipótesis interpretativas del sentido de las practicas sociales, con "referencia a la situación existencial del colonizado" (Bourdieu et al., 1963: 
258). ¿Cómo no ver allí una forma posible para la transposición sociológica del "análisis existencial" propuesto por Merleau-Ponty en la Fenomenología de la percepción, cuando señala que "no hay explicaciones sin comprensión" y propone encontrar "para el pensamiento estadístico un fundamento fenomenológico”? (cfr. Merleau-Ponty, 1945: 134, 158, 505).

La introducción de la pregunta por el sentido, punto de encuentro clave entre la fenomenología y la sociología weberiana (Schutz lo tenía muy claro), está dada, en Bourdieu, por el papel central que ocupa la enseñanza de Merleau-Ponty (cfr. Dukuen, 2010) y su impronta no sólo en la producción de una Teoría de la práctica cuyo centro es el cuerpo (Leib), sino también en el desarrollo de esta apuesta metodológica relativa a superar la oposición entre explicar y comprender, que sin dudas sería el lugar más extraño donde encontrar la impronta merleaupontyana, si no fuera por la apertura positiva — sin dejar de ser crítica - que Merleau-Ponty mostró siempre frente a las ciencias sociales.

En ese sentido, se comprende su propuesta de un diálogo entre filosofía y sociología, lo cual implica dilucidar un "intermundo" que en Las aventuras de la dialéctica Merleau-Ponty (1955) llama "verdad o historia a hacer"; esto permitiría sobrepasar la oposición entre objetivo y subjetivo, pasividad y actividad, autonomía e independencia. Si como señalamos con anterioridad, Bourdieu llega a Weber a través de este notable libro de Merleau-Ponty, sus perspectivas se entrelazan en la apuesta metodológica bourdiana relativa a superar la oposición entre explicación y comprensión que hemos descrito en este artículo, y que en términos posteriores y más amplios aparecerá en la propuesta de superación de objetivismo y subjetivismo (cfr. Bourdieu, 1972, 1980).

Este punto nos lleva al debate actual sobre la relación de la obra de Bourdieu con la fenomenología, el cual gira en torno a si es un lector más o menos fiel de esa tradición, en la reasunción y reelaboración de conceptos y problemáticas. Como hemos señalado en la introducción, si bien hay toda una serie de trabajos que muestran la antropologización de problemáticas fenomenológicas realizada por el autor (Martínez, 2007; Dukuen, 2012, 2013, entre otros) en los últimos años, Bourdieu ha recibido críticas desde perspectivas de orientación fenomenológicas por "mal interpretar" a Husserl, al no tomar en consideración la fenomenología genética (cfr. Throop y Murphy, 2002), proponer un concepto de habitus incapaz de dar cuenta del sustrato de las acciones sociales al caer en el problema de la fundamentación (cfr. Belvedere, 2011) y evaluar las obras de Sartre y Schutz mediante lecturas parciales, como subjetivistas (cfr. Throop y Murphy, 2002; Belvedere, 2012). 
Creemos que estas críticas aciertan en la parcialidad de las apreciaciones de Bourdieu sobre Husserl y Schutz y en los problemas de la teoría del habitus, cuestiones que hemos abordado y reelaborado a partir de Merleau-Ponty en otro lado (Dukuen, 2013). Respecto a Sartre, Bourdieu (1972: 248-250; 1980, cap. 2) le realiza objeciones filosóficas muy cercanas a las de MerleauPonty (1945: 496 y ss) relativas a la libertad y a la oposición entre el en-si y el para-si (cfr. Sapiro, 2007 a y b).

En ese sentido, si bien discutibles, esas objeciones no carecen de fundamento, lo cual permite comprender que un notable especialista en la obra de Merleau-Ponty, Etienne Bimbenet, ubique la propuesta de Bourdieu como "una ejemplificación sociológica fiel de la fenomenología merleaupontyana de la percepción” (Bimbenet, 2006: 58; 2011: 167). Sin embargo, las críticas de orientación fenomenológica no tienen en cuenta cuál es el sentido de la apropiación que hace Bourdieu de la fenomenología.

Por eso mismo llamamos la atención sobre la respuesta de Bourdieu (2002) al artículo crítico de Throop y Murphy (2002) sobre su relación con la fenomenología, donde indica que es necesario tener en cuenta que su retoma de problemáticas fenomenológicas no se basa en una transposición metódica, sino en una reasunción donde las ideas teóricas "están diseñadas para guiar la investigación empírica y para resolver problemas específicos de la antropología y la sociología" (Bourdieu, 2002: 209). Justamente por ello, en este trabajo hemos querido mostrar esa reasunción, centrándonos especialmente en la fundamentación desconocida que encuentra la apuesta metodológica de Bourdieu en Argelia, en la enseñanza de Merleau-Ponty. Esperamos de esta manera haber contribuido a un abordaje multidimensional de la obra de Bourdieu, que permita abrir nuevos diálogos entre las ciencias sociales y la filosofía.

\section{Bibliografía}

AAvv (2000), “Autour de Pierre Bourdieu et de l'anthropologie”, en Revue Awal. Cahier d'Études Berbères, núm. 21, París.

AAvv (2003), “L'Autre Bourdieu”, en Revue Awal. Cahier d'Études Berbères, núm. 27-28, París.

Addi, Lahouari (2002), Sociologie et anthropologie chez Pierre Bourdieu: Le paradigme anthropologique Kabyle et ses conséquences théoriques, París: Éditions La Découverte.

Baranger, Denis (2004), Epistemología y metodología en la obra de Pierre Bourdieu, Buenos Aires: Prometeo. 
Convergencia Revista de Ciencias Sociales, núm. 68, 2015, Universidad Autónoma del Estado de México

Belvedere, Carlos (2011a), "Bourdieu's concept of habitus as a substruction of the monad", en Annual Conference. Society for Phenomenology and the Human Sciences, Filadelfia, Estados Unidos.

Belvedere, Carlos (2011b), Problemas de fenomenología social. A propósito de Alfred Schutz, las ciencias sociales y las cosas mismas, Buenos Aires: Prometeo.

Belvedere, Carlos (2012), El discurso del dualismo en la teoría social contemporánea. Una critica fenomenológica, Buenos Aires: Eudeba.

Bimbenet, Étienne (2006), "Sens pratique et pratiques réflexives. Quelques développements sociologiques de l'ontologie merleau-pontienne”, en Archives de Philosophie, 2006/1, tomo 69, París.

Bimbenet, Étienne (2011), Après Merleau-Ponty: études sur la fécondité d'une pensée, París: Vrin.

Bourdieu, Pierre (1958), Sociologie de l'Algérie, París: PUF.

Bourdieu, Pierre (1960), “Guerre et mutation sociale en Algérie”, en Etudes méditerranéennes, núm. 7, París.

Bourdieu, Pierre (1961), "Revolution nans la revolution”, en Esprit, núm. 1, París.

Bourdieu, Pierre (1962a), "La hantise du chômage chez l'ouvrier algérien”, en Sociologie du travail, tomo 4, París.

Bourdieu, Pierre (1962b), “Les relaciones entre les sexes dans la société paysanne”, en Les Temps Modernes, núm. 195, París.

Bourdieu, Pierre (2002), “Célibat et condition paysanne”, en Le bal des célibataires, París: Seuil.

Bourdieu, Pierre (1963), "La société traditionnelle. Attitude à l'égard du temps et conduite économique”, en Sociologie du travail, tomo 5, París.

Bourdieu, Pierre (1966), "Condition de classe et position de classe”, en Archives européennes de sociologie, tomo VII, núm. 2, París.

Bourdieu, Pierre (1972), Esquisse d'une théorie de la pratique, précédé de trois études d'ethnologie Kabyle, Genève: Droz.

Bourdieu, Pierre (1980), Le sens pratique, París: Minuit.

Bourdieu, Pierre (1987), "Fieldwork in Philosophy", en Choses dites, París: Minuit.

Bourdieu, Pierre (1990), "La domination masculine", en Actes de la recherche en sciences sociales, vol. 84, París.

Bourdieu, Pierre (1993), La misère du monde, París: Seuil.

Bourdieu, Pierre (2003), Méditations pascaliennes, París: Seuil.

Bourdieu, Pierre (1998), La domination masculine, París: Seuil.

Bourdieu, Pierre (2006), Argelia 60. Estructuras económicas y estructuras temporales, Buenos Aires: Siglo Xx.

Bourdieu, Pierre (2000), "Las condiciones sociales de la circulación de las ideas", en Intelectuales, politica y poder, Buenos Aires: Eudeba.

Bourdieu, Pierre (2001), Science de la science et réfléxivité, París: Raisons d'agir. 
Bourdieu, Pierre (2002), "Response to Throop \& Murphy”, en Anthropological Theory, vol. 2 , núm. 2, London.

Bourdieu, Pierre (2004), Esquisse pour une auto-analyse, París: Raisons d'agir.

Bourdieu, Pierre et al. (1963), Travail et Travailleurs en Algérie, París: Mouton.

Bourdieu, Pierre y Sayad Abdelmalek (1964), Le déracinement, París: Minuit.

Bourdieu, Pierre y Jean Claude Passeron (1967), "Sociology and philosophy in France since 1945: Death and resurrection of a philosophy without a subject”, en Social Research, vol. 34, núm. 1, New York.

Bourdieu, Pierre y Jean Claude Passeron (1970), La reproduction, París: Minuit.

Bourdieu, Pierre (1971a), "Une interprétation de la théorie de la religión selon Max Weber", en Archives européennes de sociologie, vol XII, núm. 1, París.

Bourdieu, Pierre (1971b), "Genèse et structure du champ religieux", en Revue française de sociologie, vol. 12, París.

Bourdieu, Pierre, Jean Claude Chamboredon y Jean Claude Passeron (1973), Le métier de sociologue, París: Mouton Bordas.

Bourdieu, Pierre, Franz Schultheis y Andreas Pfeuffer (2011), "With Weber Against Weber: In Conversation With Pierre Bourdieu”, en Susen S. y Turner B. S., The Legacy of Pierre Bourdieu Critical Essays, UK-NY: Anthem Press.

Dukuen, Juan (2010), "Entre Schutz y Bourdieu. Encuentros y desencuentros en fenomenología social”, en Revista Latinoamericana de Estudios sobre Cuerpos, Emociones y Sociedad, núm. 3, año 2, agosto 2010, Córdoba; CEA, CONICET, UnC.

Dukuen, Juan (2011a), "Retomar un debate. La teoría de la violencia simbólica en Bourdieu y la noción de Ideología en Althusser, frente al problema de la reproducción”, en Intersticios. Revista sociológica de pensamiento crítico, vol. 5, núm. 1, Madrid.

Dukuen, Juan (2011b), “Temporalidad, habitus y violencia simbólica. Génesis de una teoría de la dominación en la obra de Bourdieu”, en Revista Avatares de la Comunicación y la Cultura, año 2, núm. 2, agosto, Fac. de Ciencias Sociales, Universidad de Buenos Aires.

Dukuen, Juan (2012), “Bourdieu. Un 'trabajo de campo' en fenomenología”, en XXIII Encuentro Nacional de Fenomenología y Hermenéutica, septiembre, Buenos Aires; Academia Nacional de Ciencias.

Dukuen, Juan (2013), Habitus y dominación. Para una critica de la teoría de la violencia simbólica en Bourdieu, Tesis de Doctorado, Facultad de Ciencias Sociales, Universidad de Buenos Aires.

Durkheim, Émile (2008), Las reglas del método sociológico, Buenos Aires: Losada.

García, Esteban (2008), "El comportamiento corporal, los poderes y las normas (M. MerleauPonty, M. Foucault, P. Bourdieu, J. Butler)”, Ponencia en Coloquio Internacional. Merleau-Ponty viviente, 3-6 de septiembre de 2008, México.

García, Esteban (2012), Maurice Merleau-Ponty. Filosofía, corporalidady percepción, Buenos Aires: Rhesis.

Grange, Juliette (2011), "El habitus, de la filosofía a la sociología, ida y vuelta”, en Pierre Bourdieu. Un filósofo de la sociología, Buenos Aires: Nueva Visión. 
Gutiérrez, Alicia Beatriz (2003), “Con Marx y contra Marx’: El materialismo en Pierre Bourdieu”, en Revista Complutense de Educación, vol. 14, núm. 2, Madrid.

Haber, S. (2004), "La sociologique française contemporaine devant le concept bourdieusien d'habitus”, en Alter. Revue de phénoménologie, núm. 12, París.

Heidegger, Martín (2002), Ser y Tiempo, Madrid: Trotta.

Heran, François (1987), "La seconde nature de l'habitus. Tradition philosophique et sens commun dans le langage sociologique", en Revue française de sociologie, vol. 28, núm. 3, París.

Hong Sung-Min (1999), Habitus, corps, domination: Sur certains presupposes philosophiques de la sociologie de Pierre Bourdieu, París: L'Harmattan.

Husserl, Edmund (1962), Ideas I, México: Fondo de Cultura Económica.

Husserl, Edmund (2002), Lecciones sobre la fenomenología de la conciencia interna del tiempo, Madrid: Trotta.

Husserl, Edmund (1980), Experiencia y Juicio, México: Universidad Nacional Autónoma de México.

Husserl, Edmund (2009), La crisis de las ciencias europeas y la fenomenología trascendental, Buenos Aires: Prometeo.

Husserl, Edmund (1997), Ideas II, México: Universidad Nacional Autónoma de México.

Karsenti, Bruno (2011), “De Marx a Bourdieu”, en De Fornell M. y A. Ogien, Bourdieu, théoricien de la pratique, París: EHEss.

Lane, Jeremy (2000), Pierre Bourdieu. A Critical Introduction, Londres: Pluto.

Martín Criado, Enrique (2006), "Estudio introductorio: las dos Argelias de Pierre Bourdieu”, en Bourdieu P., Sociología de Argelia y Tres estudios de etnología Cabilia, Madrid: CEI/BOE.

Martínez, Ana Teresa (2007), Pierre Bourdieu. Razones y lecciones de una práctica sociológica, Buenos Aires: Manantial.

Mauss, Marcel (1979), “Técnicas y movimientos corporales”, en Sociología y Antropología, Madrid: Tecnos.

Merleau-Ponty, Maurice (1942), La structure du comportament, París: Gallimard.

Merleau-Ponty, Maurice (1945), Phénoménologie de la perception, París: Gallimard.

Merleau-Ponty, Maurice (1977), La fenomenologia y las ciencias del hombre, Buenos Aires: Noya.

Merleau-Ponty, Maurice (1955), Les aventures de la dialectique, París: Gallimard.

Merleau-Ponty, Maurice (1960), "Le philosophe et la sociologie”, en Signes, París: Gallimard.

Merleau-Ponty, Maurice (1960), “De Mauss a Claude Lévi-Strauss”, en Signes, París: Gallimard.

Moreno Pestaña, José Luis (2011), “Estudio preliminar”, en Passeron J. C. (2011), El razonamiento sociológico. El espacio comparativo de las pruebas históricas, Madrid: Siglo XXI.

Nouschi, André (2003), "Autour de Sociologie de l'Algerie”, en Awall, núm. 27-28, París. 
Juan Dukuen. Una fundamentación “merleaupontyana” para la apuesta metodológica de Bourdieu en Argelia

Pinto, Louis (2007), "Voluntades de saber. Bourdieu, Derrida, Foucault", en Champagne P. et al., Pierre Bourdieu, sociólogo, Buenos Aires: Nueva Visión.

Pintos-Peñaranda, María Luz (2008), "El neuropsiquiatra Kurt Goldstein en la génesis del pensamiento fenomenológico de Merleau-Ponty”, en Investigaciones Fenomenológicas Volumen Extra Serie Monográfica 1, Sociedad Española de Fenomenología UnEd, Madrid.

Passeron, Jean Claude (2011), El razonamiento sociológico. El espacio comparativo de las pruebas históricas, Madrid: Siglo XXI.

Ralón de Walton, Graciela (2008), "El campo de la cultura: simbolismo y verdad a hacer", en Conferencia Mesa inaugural Coloquio Internacional. Merleau-Ponty viviente, septiembre, México.

Ralón de Walton, Graciela (2010), “La lógica práctica y la noción de hábito”, en Anuario Colombiano de Fenomenología, vol. IV, octubre, Bogotá, Colombia.

Ralón de Walton, Graciela y Juan Dukuen (2013), "Los modos de dominación en la socioantropología de Bourdieu. Esbozo de una crítica”, en Revista Estudios de Filosofía, núm. 47, Medellín, Colombia: Universidad de Antioquía.

Reed-Danahay, Deborah (2005), Locating Bourdieu, Bloomington e Indianapolis, Indiana: University Press.

Rey, Jean François (2011), "Hacer el tiempo. De una fenomenología de las actitudes temporales a una teoría de las prácticas temporales”, en Pierre Bourdieu. Un filósofo de la sociología, Buenos Aires: Nueva Visión.

Ricoeur, Paul (2001), “¿Qué es un texto?”, en Del texto a la acción, Buenos Aires: FCE.

Sapiro, Gisèle (2007a), “Una libertad restringida. La formación de la teoría del habitus”, en Champagne P. et al., Pierre Bourdieu, sociólogo, Buenos Aires: Nueva Visión.

Sapiro, Gisèle (2007b), "Entrevista sobre la fenomenología”, en Champagne P. et al., Pierre Bourdieu, sociólogo, Buenos Aires: Nueva Visión.

Sartre, Jean Paul (1960), "Questions de méthode”, en Critique de la raison dialectique, tomo I, París: Gallimard.

Schutz, Alfred (1993), La construcción significativa del mundo social, Barcelona: Paidós.

Throop, Jason y Keith Murphy (2002), "Bourdieu and Phenomenology: A Critical Assessment”, en Anthropological Theory, vol. 2, núm. 2, London.

Vázquez García, Francisco (2002), Pierre Bourdieu, la sociología como crítica de la razón, Barcelona: Montesinos.

Wacqüant, Loïc (1992), “Introduction”, en Bourdieu, P. y L. Wacqüant, Réponses, París: Seuil.

Wacqüant, Loïc (2001), “Further notes on Bourdieu's 'Marxism”, en International Journal of Contemporany Sociology, núm. 38, Auburn.

Weber, Max (2007), Economía y Sociedad, México: Fondo de Cultura Económica.

Wright Mills, Charles (1961), La imaginación sociológica, México: Fondo de Cultura Económica. 
Juan Dukuen. Doctor en Ciencias Sociales, Universidad de Buenos Aires. Becario Postdoctoral Conicet-Argentina y docente de la Universidad de Buenos Aires. Investiga el vínculo entre la socioantropología de Bourdieu y la fenomenología de Merleau-Ponty y sus potencialidades en la investigación empírica de la formación de disposiciones políticas en jóvenes escolarizados de clase alta. Participó en diversos congresos sobre teoría sociológica, antropología y filosofía. Publicaciones recientes: Juan Dukuen, Las astucias del poder simbólico, Buenos Aires: Koyatún (2010); "Otros territorios: una discusión sobre la relación entre cultura y política desde Bourdieu aplicable al estudio de jóvenes escolarizados", en Revista Argentina de Estudios de Juventud, núm. 7, diciembre, Argentina: Universidad Nacional de La Plata (2013); con Graciela Ralón de Walton, "Los modos de dominación en la socio-antropología de Bourdieu. Esbozo de una crítica”, en Revista Estudios de Filosofía, núm. 47, junio, Medellín, Colombia (2013).

Recepción: 20 de marzo de 2014.

Aprobación: 18 de diciembre de 2014. 